\title{
REES MATRIX SEMIGROUPS
}

\author{
by MARK V. LAWSON*
}

(Received 16th November 1987; revised 20th March 1989)

\begin{abstract}
In this paper we provide a new, abstract characterisation of classical Rees matrix semigroups over monoids with zero. The corresponding abstract class of semigroups is obtained by abstracting a number of algebraic properties from completely 0-simple semigroups: in particular, the relationship between arbitrary elements and idempotents.
\end{abstract}

1980 Mathematics subject classification (1985 Revision): $20 \mathrm{M} 10$.

\section{Introduction}

The aim of this paper is to prove a new, abstract characterisation of Rees matrix semigroups over monoids having regular sandwich matrices.

The motivation for wanting such characterisations is not hard to provide: from their introduction in the fundamental paper of Rees [15], building on the pioneering work of Suschkevitch [17], Rees matrix semigroups have established themselves as one of the most useful semigroup theoretic constructions - as a glance at Meakin's survey article will verify [14]. Their usefulness lies in providing a technique for constructing new classes of semigroups from known or simpler classes. The Rees Theorem itself constructs completely 0-simple semigroups via the Rees construction from groups (see Howie [6, Theorem III 2.5]).

In looking for abstract characterisations of Rees matrix semigroups, it is natural to take some defining characteristic of completely 0 -simple semigroups and then by generalisation show how it describes abstractly a class of Rees matrix semigroups. The two properties (i) and (ii) below were the starting points for abstract characterisations of Rees matrix semigroups by Steinfeld [16] and Lallement and Petrich [7] respectively:

(i) (Proposition 3.4 [16].) A semigroup $S$ with zero is completely 0 -simple iff $S$ has the form $S=\bigcup_{\lambda \in \Lambda} S e_{\lambda}\left(e_{\lambda}^{2}=e_{\lambda}\right)$ where the $S e_{\lambda}$ are pairwise left $S$-similar 0 -minimal left ideals of $S$.

(ii) (Theorem 4.5 [7].) A semigroup $S$ with zero is completely 0 -simple iff $S$ is regular, (0) is "matriciel" in $S$ and the non-zero classes of the finest "0-matricielle" congruence are groups.

*This work was supported by a Junior Research Fellowship at Lincoln College, Oxford. 
Steinfeld generalised (i) to give an abstract characterisation of the class of Rees matrix semigroups over monoids with zero having locally regular sandwich matrices. Lallement and Petrich generalising (ii) characterised Rees matrix semigroups over monoids with zero adjoined, the sandwich matrix being regular over the group of units of the monoid.

There is, however, a third well-known characterisation:

(iii) (Howie [6, Theorem III 3.5].) A semigroup $S$ with zero is completely 0-simple iff $S$ is a 0 -bisimple, regular semigroup in which every non-zero idempotent is primitive.

Our aim is to characterise Rees matrix semigroups by generalising (iii). We achieve this by generalising, in the first instance, the class of regular semigroups to a class we have dubbed " $U$-semiabundant". The origin of this class of semigroups lies in the thesis of El-Qallali [4] and a paper of de Barros [2]: the general theory of such semigroups is pursued in detail in [11] and [12].

The paper is divided into three sections. In Section 1, we show that a Rees matrix semigroup has a number of important properties with respect to a distinguished subset of its idempotents. Sections 2 and 3 are dedicated to showing that these properties characterise Rees matrix semigroups; Section 2 introduces the class of $U$-semiabundant semigroups and in particular the primitive $U$-semiabundant semigroups and we obtain a number of structural results for this class of semigroups generalising work by Fountain [5]; in Section 3 we specialise down to a class of primitive $U$-semiabundant semigroups which we call "Rees semigroups"-these, we show, may be coordinatised by Rees matrix semigroups, obtaining the converse results to those of Section 1. Finally we mention that the results of this paper may be generalised to incorporate those of Steinfeld [16]-where Rees matrix semigroups with locally regular sandwich matrices are considered.

In a subsequent paper [10] we will extend the work of Fountain [5] to obtain a description of a class of blocked Rees matrix semigroups.

\section{Rees matrix semigroups}

Let $S$ be a monoid with identity 1 and zero element 0 , having group of units $G(S)$. Let $\Lambda$ and $I$ be non-empty sets and let $P$ be a $\Lambda \times I$-matrix over $S$ with entries $p_{\lambda i}$ where $(\lambda, i) \in \Lambda \times I$. The Rees matrix semigroup $M=M^{0}(S ; I, \Lambda ; P)$ is the set of triples $I \times S \times \Lambda$ with a zero element 0 adjoined and where we identify all the elements of the form $(i, 0, \lambda)$ with 0 , under a multiplication given by

$$
(i, x, \lambda)(j, y, \mu)=\left\{\begin{array}{l}
\left(i, x p_{\lambda j} y, \mu\right) \text { if } p_{\lambda j} \neq 0 \\
0 \text { otherwise. }
\end{array}\right.
$$

The matrix $P$ is called regular if each row and each column contains an element from $G(S)$. From now on all Rees matrix semigroups will be assumed to be over a monoid 
and to have regular sandwich matrices. If $a \in G(S)$ we will write $a^{-1}$ for its group inverse. We will denote the set of generalised inverses of the element $a$ by $V(a)$, and we will write $a^{\prime} \in V(a)$. The set of all idempotents of a Rees matrix semigroup will be denoted by $E$ or $E(M)$.

Define the set of projections of $M$ to be the following,

$$
P(M)=\left\{(i, x, \lambda): p_{\lambda i} \in G(S) \text { and } x=p_{\lambda i}^{-1}\right\} \cup\{0\}
$$

Lemma 1.1. The projections are idempotents and every element of $M$ has a right and left identity in $P(M)$.

Proof. If $\left(i, p_{\lambda i}^{-1}, \lambda\right) \in P(M) \quad$ then $\quad\left(i, p_{\lambda i}^{-1}, \lambda\right)^{2}=\left(i, p_{\lambda i}^{-1} p_{\lambda i} p_{\lambda i}^{-1}, \lambda\right)=\left(i, p_{\lambda i}^{-1}, \lambda\right) \quad$ Let $(i, x, \lambda) \in M$. Since $P$ is regular there exists an invertible element $p_{\lambda i(\lambda)}$ for some $i(\lambda) \in I$ and similarly an invertible $p_{\lambda(i) i}$ for some $\lambda(i) \in \Lambda$. But then $(i, x, \lambda)=(i, x, \lambda)(i(\lambda)$, $\left.p_{\lambda i(\lambda)}^{-1}, \lambda\right)=\left(i, p_{\lambda(i) i}^{-1}, \lambda(i)\right)(i, x, \lambda)$.

Lemma 1.2. If $\left(i, p_{\lambda i}^{-1}, \lambda\right) \in P(M)$ then,

(i) $(j, x, \mu)\left(i, p_{\lambda i}^{-1}, \lambda\right)=(j, x, \mu)$ iff $\lambda=\mu$.

(ii) $\left(i, p_{\lambda i}^{-1}, \lambda\right)(j, x, \mu)=(j, x, \mu)$ iff $i=j$.

Proof. We will prove case (i), case (ii) follows similarly. If $(j, x, \mu)\left(i, p_{\lambda i}^{-1}, \lambda\right)=(j, x, \mu)$ then $\left(j, x p_{\mu i} p_{\lambda i}^{-1}, \lambda\right)=(j, x, \mu)$ which implies $\lambda=\mu$.

Conversely, if $\lambda=\mu$ then since $p_{\mu i} p_{\mu i}^{-1}=1$ we have $x p_{\mu i} p_{\mu i}^{-1}=x$ so that $(j, x, \mu)\left(i, p_{\lambda i}^{-1}, \lambda\right)=(j, x, \mu)$.

It is important to note that in general $P(M)$ is a proper subset of $E(S)$. For the following result we use the (non-standard) notation

$$
R(x)=\{a \in S: a x a=a\}
$$

Lemma 1.3. $P(M)=E(M)$ iff $\cup\left\{R\left(p_{\lambda i}\right):(\lambda, i) \in \Lambda \times I\right) \subseteq G(S)^{0}$.

Proof. Note that $(i, x, \lambda) \in E(M)$ iff $x=0$ or $x=x p_{\lambda i} x$ that is $x \in R\left(p_{\lambda i}\right)$. Let $P(M)=$ $E(M)$. Let $x \neq 0$ and $x \in \cup\left\{R\left(p_{\lambda i}\right):(\lambda, i) \in \Lambda \times I\right\}$. Then $x=x p_{\lambda i} x$ for some $(\lambda, i) \in \Lambda \times I$. But then $(i, x, \lambda) \in E(M)$ so that $(i, x, \lambda) \in P(M)$ by assumption, giving $x=p_{\lambda i}^{-1}$. Thus $x \in G(S)$.

Conversely, let $\cup\left\{R\left(p_{\lambda i}\right\}:(\lambda, i) \in \Lambda \times I\right) \subseteq G(S)^{0}$ and let $(i, x, \lambda) \in E(M) \backslash\{0\}$. Then $x \in R\left(p_{\lambda i}\right)$ so that $x \in G(S)$ but then $x=x p_{\lambda i} x$ implies $x p_{\lambda i}=p_{\lambda i} x=1$ giving $x=p_{\lambda i}^{-1}$ whence $(i, x, \lambda) \in P(M)$.

A unipotent monoid is a monoid with a unique idempotent. It is easy to see that in such monoids regular elements are invertible. The following is now immediate:

Corollary 1.4. If $S$ is a unipotent monoid then $P(M)=E(M)$. 
The previous corollary holds, in particular, for groups and cancellative monoids.

Define two relations $\tilde{L}$ and $\tilde{R}$ on $M$ as follows:

$$
\begin{gathered}
(i, x, \lambda) \tilde{L}(j, y, \mu) \text { iff (for all } \mathbf{e} \in P)((i, x, \lambda) \mathbf{e}=(i, x, \lambda) \text { iff }(j, y, \mu) \mathbf{e}=(j, y, \mu)) \\
(i, x, \lambda) \tilde{R}(j, y, \mu) \text { iff (for all } \mathbf{e} \in P)(\mathbf{e}(i, x, \lambda)=(i, x, \lambda) \text { iff } \mathbf{e}(j, y, \mu)=(j, y, \mu))
\end{gathered}
$$

It is clear that $\tilde{L}$ and $\tilde{R}$ are equivalence relations on $M$. In addition, define the equivalences $\tilde{H}=\tilde{L} \wedge \tilde{R}$ and $\tilde{D}=\tilde{L} \vee \tilde{R}$.

Proposition 1.5. If $(i, x, \lambda),(j, y, \mu) \in M$ then:

(i) $(i, x, \lambda) \tilde{L}(j, y, \mu)$ iff $(i, x, \lambda)=(j, y, \mu)=0$ or both elements are non-zero and $\lambda=\mu$.

(ii) $(i, x, \lambda) \tilde{R}(j, y, \mu)$ iff $(i, x, \lambda)=(j, y, \mu)=0$ or both elements are non-zero and $i=j$.

(iii) If $(\mathbf{a}, \mathbf{b}) \in \tilde{L}$ and $\mathbf{x} \in M$ and $\mathbf{a x}, \mathbf{b x} \neq \mathbf{0}$ then $(\mathbf{a x}, \mathbf{b x}) \in \tilde{L}$.

(iv) If $(\mathbf{a}, \mathbf{b}) \in \tilde{R}$ and $\mathbf{x} \in M$ and $\mathbf{x a}, \mathbf{x b} \neq \mathbf{0}$ then $(\mathbf{x a}, \mathbf{x b}) \in \tilde{R}$.

(v) Each $\tilde{L}$-class and each $\tilde{R}$-class contains at least one projection.

Proof. (i) By Lemma 1.2 it is clear that if $(i, x, \lambda),(j, y, \mu) \neq 0$ then

$$
(i, x, \lambda) \tilde{L}(j, y, \mu) \text { iff } \lambda=\mu \text {. }
$$

Conversely, if $(i, x, \lambda)=0$ then $(i, x, \lambda)=(i, 0, \mu)$. Since $0^{2}=0$ and $\mathbf{0} \in P(M)$ and given that $(i, x, \lambda) \tilde{L}(j, y, \mu)$ we must have that $(j, y, \mu) 0=(j, y, \mu)$. But this implies $(j, y, \mu)=0$. The proof of (ii) is similar.

(iii) Suppose that $(i, x, \lambda),(j, y, \lambda) \neq \mathbf{0}$ and $(i, x, \dot{\lambda}) \tilde{L}(j, y, \lambda)$. If $(k, z, \mu) \in M$ then,

$$
\begin{aligned}
& (i, x, \lambda)(k, z, \mu)=\left(i, x p_{\lambda k} z, \mu\right) \\
& (j, y, \lambda)(k, z, \mu)=\left(j, y p_{\lambda k} z, \mu\right) .
\end{aligned}
$$

If $x p_{\lambda k} z, y p_{\lambda k} z \neq 0$ then $\left(i, x p_{\lambda k} z, \mu\right) \tilde{L}\left(j, y p_{\lambda k} z, \mu\right)$ by (i) above.

The proof of (iv) is similar.

(v) By the regularity of $P$ (for each $\lambda \in \Lambda$ ) (there exists $i(\lambda) \in I$ ) such that $\left(i(\lambda), p_{\lambda i(\lambda)}^{-1}, \lambda\right) \in P(M)$ thus $(i, x, \lambda) \tilde{L}\left(i(\lambda), p_{\lambda i(\lambda)}^{-1}, \lambda\right)$.

We may similarly show that each $\widetilde{R}$-class contains a projection.

Corollary 1.6. If $S$ is such that for all $x, y \in S, x y=0$ implies $x=0$ or $y=0$ then $M=M^{0}(S ; 1, \Lambda ; P)$ has the following property:

$$
\text { for } \mathbf{a}, \mathbf{b} \neq 0,(\mathbf{a}, \mathbf{b}) \in \tilde{L} \text { and } \mathbf{x} \in M \text { then } \mathbf{a x}=\mathbf{0} \text { iff } \mathbf{b x}=\mathbf{0} \text {. }
$$

Thus $\tilde{L}$ is a right congruence. Similarly $\tilde{R}$ is a left congruence. 
Proof. We consider the calculation carried out in Proposition 1.5(iii). If $\left(i, x p_{\lambda k} z, \mu\right)=$ 0 then $x p_{\lambda k} z=0$ in $S$. But under our assumption on $S$ this occurs iff $x=0$ or $p_{\lambda k}=0$ or $z=0$. By assumption $x \neq 0$ so that either $p_{\lambda k}=0$ or $z=0$ but in each case this implies $\left(j, y p_{\lambda k} z, \mu\right)=0$. That $\tilde{L}$ is a right congruence now follows from Proposition 1.5(iii).

We will now turn to look more closely at the properties of the set of projections.

If $S$ is any semigroup define preorders $\omega^{r}$ and $\omega^{l}$ on $E(S)$ by

$$
\begin{aligned}
& e \omega^{r} f \text { iff } f e=e \\
& e \omega^{l} f \text { iff } e f=e .
\end{aligned}
$$

If $P \subseteq E(S)$ we will say that $P$ is closed under basic products if $e, f \in P$ and $(e, f) \in\left(\omega^{r} \cup \omega^{l}\right) \cup\left(\omega^{r} \cup \omega^{l}\right)^{-1}$ implies $e f \in P$. Note that to prove that a subset $P$ is closed under basic products it is enough to show that for $e, f \in P$

$$
(e, f) \in \omega^{r} \text { implies } e f \in P \text { and }(e, f) \in \omega^{l} \text { implies } f e \in P \text {. }
$$

\section{Proposition 1.7.}

(i) The set $P(M) \backslash\{0\}$ is totally unordered under $\omega$.

(ii) The set $P(M)$ is closed under basic products.

(iii) The elements of $P(M) \backslash\{0\}$ are all D-related.

Proof. (i) Let $\left(i, p_{\lambda i}^{-1}, \lambda\right), \quad\left(j, p_{\mu j}^{-1}, \mu\right) \in P(M) \backslash\{0\} \quad$ with $\quad\left(i, p_{\lambda i}^{-1}, \lambda\right) \omega\left(j, p_{\mu j}^{-1}, \mu\right)$. Then $\left(j, p_{\mu j}^{-1}, \mu\right)$ is a left and right identity for $\left(i, p_{\lambda i}^{-1}, \lambda\right)$ so that by Lemma $1.2, i=j$, and $\lambda=\mu$, giving $p_{\lambda i}^{-1}=p_{\mu j}^{-1}$.

(ii) If $\left(j, p_{\mu j}^{-1}, \mu\right) \omega^{r}\left(i, p_{\lambda i}^{-1}, \lambda\right)$ then $\left(i, p_{\lambda i}^{-1}, \lambda\right)$ is a left identity for $\left(j, p_{\mu j}^{-1}, \lambda\right)$ so that by Lemma 1.2 we have $i=j$. Thus, $\left(i, p_{\mu i}^{-1}, \mu\right)\left(i, p_{\lambda i}^{-1}, \lambda\right)=\left(i, p_{\mu i}^{-1} p_{\mu i} p_{\lambda i}^{-1}, \lambda\right)=\left(i, p_{\lambda i}^{-1}, \lambda\right) \in P(M)$. A similar result holds for $\omega^{t}$.

(iii) Let $\left(i, p_{\lambda i}^{-1}, \lambda\right),\left(j, p_{\mu j}^{-1}, \mu\right) \in P(M) \backslash\{0\}$. Consider the elements $\left(i, p_{\lambda i}^{-1}, \mu\right)$ and $\left(j, p_{\mu j}^{-1}, \lambda\right)$. It is easy to check that $\left(i, p_{\lambda i}^{-1}, \mu\right) \in V\left(\left(j, p_{\mu j}^{-1}, \lambda\right)\right)$. Also, $\left(i, p_{\lambda i}^{-1}, \mu\right)\left(j, p_{\mu j}^{-1}, \lambda\right)=$ $\left(i, p_{\lambda i}^{-1}, \lambda\right)$ and $\left(j, p_{\mu j}^{-1}, \lambda\right)\left(i, p_{\lambda i}^{-1}, \mu\right)=\left(j, p_{\mu j}^{-1}, \mu\right)$. Now apply Proposition II 3.6 of Howie $[6]$.

The next result shows that local submonoids with respect to projections are of interest.

Lemma 1.8. If $\mathrm{e} \in P(M), \mathrm{e} \neq 0$ then $\mathrm{e} M \mathrm{e} \simeq S$.

Proof. Let $\mathrm{e}=\left(i, p_{\lambda i}^{-1}, \lambda\right)$ and define a map $\theta: S \rightarrow \mathrm{e} M \mathrm{e}$ by $\theta(a)=\left(i, a p_{\lambda i}^{-1}, \lambda\right)$. It is easy to check that this is an isomorphism. 
Lemma 1.9. The semigroup $M=M^{0}(S ; I, \Lambda ; P)$ has the property that $\tilde{L}$ is a right congruence and $\tilde{R}$ is a left congruence iff for all $x, y \in S, x y=0$ implies $x=0$ or $y=0$.

Proof. $(\leftarrow)$ By Corollary 1.6.

$(\rightarrow)$ Let $x y=0$ and $p_{\lambda i} \in G(S)$ for some $(\lambda, i) \in \Lambda \times I$. Then using the notation of Lemma 1.8 ,

$$
\theta(x)=\left(i, x p_{\lambda i}^{-1}, \lambda\right) \text { and } \theta(y)=\left(i, y p_{\lambda i}^{-1}, \lambda\right)
$$

and $S \simeq\left(i, p_{\lambda i}^{-1}, \lambda\right) M\left(i, p_{\lambda i}^{-1}, \lambda\right)$.

Now $x=0$ iff $x p_{\lambda i}^{-1}=0$ and $y=0$ iff $y p_{\lambda i}^{-1}=0$. Either $\left(i, x p_{\lambda i}^{-1}, \lambda\right)=0=\left(i, y p_{\lambda i}^{-1}, \lambda\right)$ in which case $x=y=0$ or at least one is non-zero, say $\left(i, x p_{\lambda i}^{-1}, \lambda\right) \neq 0$. Then $\left(i, x p_{\lambda i}^{-1}, \lambda\right) \tilde{L}\left(i, p_{\lambda i}^{-1}, \lambda\right)$. But by assumption $\tilde{L}$ is a right congruence so that

$$
\left(i, x p_{\lambda i}^{-1}, \lambda\right)\left(i, y p_{\lambda i}^{-1}, \lambda\right) \tilde{L}\left(i, p_{\lambda i}^{-1}, \lambda\right)\left(i, y p_{\lambda i}^{-1}, \lambda\right)
$$

thus $\left(i, x y p_{\lambda i}^{-1}, \lambda\right) \tilde{L}\left(i, y p_{\lambda i}^{-1}, \lambda\right)$. But $\left(i, x y p_{\lambda i}^{-1}, \lambda\right)=0$ so that $0 \tilde{L}\left(i, y p_{\lambda i}^{-1}, \lambda\right)$. This implies $y p_{\lambda i}^{-1}=0$ giving $y=0$.

\section{2. $U$-semiabundant semigroups}

We will now begin the process of showing how the properties we derived for $M$ in the last section may be used to characterise $M$. Let $S$ be a semigroup with zero and let $U \subseteq E \subseteq S$ with $0 \in U$. Define relations $\tilde{L}$ and $\tilde{R}$ on $S$ depending on $U$ as follows:

$$
\begin{aligned}
& (a, b) \in \tilde{L} \text { iff (for all } e \in U)(a e=a \text { iff } b e=b) \\
& (a, b) \in \tilde{R} \text { iff (for all } e \in U)(e a=a \text { iff } e b=b)
\end{aligned}
$$

It is clear that $\tilde{L}$ and $\tilde{R}$ are equivalences on $S$. We will see later that these generalise the relations defined in Section 1. It is straightforward to show that $L \subseteq \tilde{L}$ and $R \subseteq \tilde{R}$, where $L$ and $R$ are the usual Green's relations.

The semigroup $S$ is called $U$-semiabundant if each $\tilde{L}$-class and each $\tilde{R}$-class contains an element from $U$. The $\tilde{L}$ (resp. $\tilde{R}$ ) equivalence class containing the element $a$ will be denoted by $\tilde{L}_{a}$ (respectively $\tilde{R}_{a}$ ). It will be convenient on occasion to denote an element of $U\left(\tilde{L}_{a}\right)$ by $a^{*}$ and an element of $U\left(\tilde{R}_{a}\right)$ by $a \dagger$ but it is important to note that these elements are not generally unique. Define in addition the equivalences:

$$
\tilde{H}=\tilde{L} \wedge \tilde{R} \text { and } \tilde{D}=\tilde{L} \vee \tilde{R} \text {. }
$$

A $U$-semiabundant semigroup is called primitive if $\omega$ restricts to equality on $U \backslash\{0\}$. We will call a semigroup $S$ a Rees semigroup if it satisfies the following conditions:

(i) $S$ is a primitive $U$-semiabundant semigroup. 
(ii) The set $U$ is closed under basic products.

(iii) $U \backslash\{0\}$ is contained in a single $D$-class of $S$.

We will show that Rees semigroups are precisely the abstract counterparts of Rees matrix semigroups.

Note. In this section we will often prove results which fall naturally into two parts-a "left" and a "right". For the most part we will only state and prove one of the versions, the statement and proof of the other will always follow by interchanging left and right.

We now turn to look at the relationship between the relations $\tilde{L}$ and $\tilde{R}$ and certain ideals of $S$. For the time being $S$ will be a semigroup with a fixed subset $U$ of $E(S)$. A right ideal $I$ of $S$ is called a $U$-admissible right ideal if for each $a \in I$ we have $\widetilde{R}_{a} \subseteq I$. Similarly we say that $I$ is a $U$-admissible left ideal if for each $a \in I$ we have $\tilde{L}_{a} \subseteq I$.

In the following lemmas we generalise some results from the theory of abundant semigroups [5].

Lemma 2.1. The intersection of any family of $U$-admissible right ideals $\left\{I_{\alpha}: \alpha \in J\right\}$ is either empty or a $U$-admissible right ideal.

Proof. Straightforward.

If $a \in S$ we define the principal $U$-admissible right ideal containing $a$, denoted by $\tilde{R}(a)$, to be the intersection of all $U$-admissible right ideals containing $a$. Likewise define $\tilde{L}(a)$ to be the intersection of all $U$-admissible left ideals containing $a$. A useful description of $\tilde{R}(a)$ and $\tilde{L}(a)$ may be obtained as follows:

Lemma 2.2. (i) $b \in \tilde{R}(a)$ iff there exist elements $a_{0}, \ldots, a_{n} \in S^{1}$ and elements $x_{1}, \ldots, x_{n} \in S$ such that $a=a_{0}$ and $b=a_{n}$ and $\left(a_{i}, a_{i-1} x_{i}\right) \in \tilde{R}$ for $i=1, \ldots, n$.

(ii) $b \in \tilde{L}(a)$ iff there exist elements $a_{0}, \ldots, a_{n} \in S^{1}$ and elements $x_{1}, \ldots, x_{n} \in S$ such that $a=a_{0}$ and $b=a_{n}$ and $\left(a_{i}, x_{i} a_{i-1}\right) \in \tilde{L}$ for $i=1, \ldots, n$.

Proof. We prove (i) the proof of (ii) is similar. Put $I=\{b \in S: b$ satisfies the conditions of the lemma $\}$. Now, $b \in I$ implies there exist $a_{0}, \ldots, a_{n} \in S$ and $x_{1}, \ldots, x_{n} \in S^{1}$ such that $a=a_{0}, b=a_{n}$ and $\left(a_{i}, a_{i-1} x_{i}\right) \in \tilde{R}$ for $i=1, \ldots, n$. If $a_{i-1} \in \tilde{R}(a)$ then $a_{i-1} x_{i} \in \tilde{R}(a)$ since $\tilde{R}(a)$ is a right ideal. But then $a_{i} \in \tilde{R}(a)$ since $\tilde{R}(a)$ is a $U$-admissible ideal. Since $a_{0}=a \in \tilde{R}(a)$ we see that $a_{i} \in \tilde{R}(a)$ for $i=0, \ldots, n$, in particular $b \in \tilde{R}(a)$ and so $I \subseteq \tilde{R}(a)$. If $b \in I$ then $\tilde{R}_{b} \subseteq I$, for if $x \tilde{R} b$ then $(x, b 1) \in \tilde{R}$. Also, if $s \in S$ then $b s \in I$ since $(b s, b s) \in \tilde{R}$. Thus $I$ is a $U$-admissible right ideal. Since $a \in I$ we thus have $\tilde{R}(a)=I$.

Corollary 2.3. (i) $a \tilde{L} b$ iff $\tilde{L}(a)=\tilde{L}(b)$.

(ii) $a \tilde{R} b$ iff $\tilde{R}(a)=\tilde{R}(b)$. 
Proof. We will prove case (i). If $(a, b) \in \tilde{L}$ it is clear that $\tilde{L}(a)=\tilde{L}(b)$. Conversely, suppose that $\tilde{L}(a)=\tilde{L}(b)$. Then $b \in \tilde{L}(a)$ so that we may find elements, $a_{0}, \ldots, a_{n} \in S$ and $x_{1}, \ldots, x_{n} \in S^{1}$ such that, $\left(a_{i}, x_{i} a_{i-1}\right) \in \tilde{L}$ for $i=1$ to $n$ where $a=a_{0}$ and $b=a_{n}$. Let $e \in U$ with $a e=a$. Then $\left(x_{1} a\right) e=x_{1} a$. But $x_{1} a L a_{1}$ gives $a_{1} e=a_{1}$. Continuing in this way we obtain $a_{n} e=a_{n}$ that is $b e=b$. We may similarly show that $b e=b$ implies $a e=a$, so that $a \tilde{L} b$.

Lemma 2.4. Let $U \subseteq E(S)$. Then if $e \in U$ the set $S e$ is a $U$-admissible left ideal and $e S$ is a $U$-admissible right ideal.

Proof. It is clear that $S e$ is a left ideal containing $e$. Let $x \in S e$ and let $x \bar{L} y$. We have $x=s e$ for some $s \in S$ so that $x e=x$. But then $y e=y$ since $x \tilde{L} y$, so that $y=y e \in S e$.

Corollary 2.5. The semigroup $S$ is U-semiabundant iff for each aES there exist elements $e, f \in U(S)$ such that,

$$
\tilde{L}(a)=S e \text { and } \tilde{R}(a)=f S .
$$

Proof. Suppose that $S$ is $U$-semiabundant. Then $a \tilde{L} e$ for some element $e \in U$. Since $a e=a$ we have $a \in S e$. But $\tilde{L}(a)$ is a left ideal and $e \in \tilde{L}(a)$ so that $S e \subseteq \tilde{L}(a)$. But $S e$ is a $U$ admissible left ideal containing the element $a$ whence $S e=\tilde{L}(a)$.

Conversely, suppose that for all $a \in S$ there exists $e, f \in U$ such that $\tilde{L}(a)=S e$ and $\tilde{R}(a)=f S$. Now $e \in S e$ and $S e$ is a $U$-admissible left ideal so that $\tilde{L}(e) \subseteq S e$. But from Se $\subseteq \tilde{L}(e)$ we have $\tilde{L}(e)=S e$. This means $\tilde{L}(a)=\tilde{L}(e)$ whence $a \tilde{L} e$ by Corollary 2.3 .

Corollary 2.6. Let $a \in S$. Then for $e \in U$ we have $(a, e) \in \tilde{L}$ iff $a \in S e$ and $S e$ is contained in every left ideal containing $a$, which is generated by an element of $U$.

Proof. Let $(a, e) \in \tilde{L}$. Then $a e=a$ giving $a \in S e$. Now let $a \in S f$ where $f \in U$. Then $a f=a$ so that $e f=e$ whence $S e \subseteq S f$.

Conversely, let $a \in S e$ where $e \in U$ and for each $f \in U$ with $a \in S f$ we have $S e \subseteq S f$. Then $\tilde{L}(a) \subseteq S e$ since $S e$ is a $U$-admissible left ideal. Since $S$ is $U$-semiabundant, by Corollary $2.5, \tilde{L}(a)=S f$ for some $f \in U$. But then $S e \subseteq S f=\tilde{L}(a)$ so that $S e=S f$ giving $(a, e) \in \tilde{L}$.

From now on $S$ will be a primitive $U$-semiabundant semigroup in which $U$ is closed under basic products.

Lemma 2.7. If $e, f \in U$ and $e S \subseteq f S$ then either $e=0$ or $e S=f S$.

Proof. From $e S \subseteq f S$ we have $e \in f S$ so that $f e=e$ giving $e \omega^{\prime} f$. By the closure of $U$ under basic products ef $\in U$. But $e f \omega f$, so that by $U$-primitivity either $e f=0$ or $e f=f$. If $e f=0$ then $e=e^{2}=f e f e=0$. If $e f=f$ then $e R f$ so that $e S=f S$.

Corollary 2.8 If $e \neq 0$ where $e \in U$ then $a \tilde{R} e$ iff $a \neq 0$ and $a \in e S$.

Proof. Suppose that $a \tilde{R} e$ then $e a=a$ so that $a \in e S$. Conversely, suppose that $a \in e S$. 
Now $a \tilde{R} f$ for some $f \in U$ so that $a \in f S$ and $f S$ is contained in any right ideal generated by an element of $U$ containing $a$. Whence $f S \subseteq e S$. By Lemma 2.7 either $f=0$ which gives $a=0$ contrary to our assumption or $f S=e S$ whence $f R e$ giving $a \tilde{R} e$, where we use the fact that $R \subseteq \tilde{R}$.

The following is immediate:

Corollary 2.9. With $S$ as above the $\tilde{R}$-classes are all of the form $e S \backslash\{0\}$ for $e \in U$.

We say that a $U$-semiabundant semigroup satisfies the congruence condition if $\tilde{L}$ and $\tilde{R}$ are right and left congruences respectively and that a $U$-semiabundant semigroup $S$ with $0 \in U$ satisfies the weak congruence condition if for all $x \in S$,

$$
(a, b) \in \tilde{L} \text { implies that if } a x \neq 0 \text { and } b x \neq 0 \text { then }(a x, b x) \in \tilde{L}
$$

together with the left-right dual for $\tilde{R}$.

Define the relations $r$ and $l$ by

$$
\begin{aligned}
& (a, b) \in r \text { iff }(a x=0 \text { iff } b x=0) \\
& (a, b) \in l \text { iff }(x a=0 \text { iff } x b=0) .
\end{aligned}
$$

Lemma 2.10. The congruence condition holds iff the weak congruence condition holds and $\tilde{L} \subseteq r$ and $\tilde{R} \subseteq l$.

Proof. Let $\tilde{L}$ be a right congruence and let $(a, b) \in \tilde{L}$. Suppose that $a x=0$. Then $a x \tilde{L} b x$ but $0 \tilde{L} b x$ gives $b x=0$. A similar argument shows that $b x=0$ implies $a x=0$. Thus $(a, b) \in 1$.

Conversely, let $(a, b) \in \tilde{L}$. Then $a x=0$ iff $b x=0$ since $\tilde{L} \subseteq r$ so if $a x=0$ then $b x=0$ and $a x L b x$. If $a x \neq 0$ then $b x \neq 0$ so that $(a x, b x) \in \tilde{L}$ by the weak congruence condition.

Lemma 2.11. Primitive $U$-semiabundant semigroups in which $U$ is closed under basic products satisfy the weak congruence condition.

Proof. If $a \tilde{L} b$ then we need to show that if $a c, b c \neq 0$ then $a c \tilde{L} b c$ for any $c \in S$. Let $c$ be $\tilde{L}$-related to $f$ where $f \in U$ so that $\tilde{L}_{c}=S f \backslash\{0\}$ by Corollary 2.9. Since $a c \neq 0$ and $b c \neq 0$ we have $a c, b c \in S f \backslash\{0\}$. But then $a c, b c \in \tilde{L}_{c}$ whence $a c \tilde{L} b c$.

Lemma 2.12. If $x, y \in S \backslash\{0\}$ and $x y \neq 0$ then $x y \in \tilde{R}_{x \dagger} \cap \tilde{L}_{y^{*}}$.

Proof. Choose $x \dagger, y^{*}$ then $x \in x \dagger S \backslash\{0\}$ by Corollary 2.9 so that $x y \in x \dagger S \cap S y^{*} \backslash\{0\}$ that is $x y \in \tilde{R}_{x \dagger} \cap \tilde{L}_{y^{*}}$.

We conclude with a result which is somewhat tangential but nevertheless of interest. 
Proposition 2.13. Let $S$ be a primitive $U$-semiabundant semigroup with $U$ closed under basic products and satisfying the congruence condition. Then:

(i) If $x, y \in S \backslash\{0\}$ choose $x \dagger, y^{*}$ then $y x=0$ iff $y^{*} x \dagger=0$.

(ii) $\tilde{H}$ is a congruence.

(iii) $\tilde{H}_{a}$ is either a subsemigroup or $\tilde{H}_{a} \tilde{H}_{a}=\{0\}$.

Proof. (i) If $x, y \in S \backslash\{0\}$ then $y^{*} x \dagger=0$ implies $y\left(y^{*} x \dagger\right) x=0$ so that $y x=0$. Conversely, let $y x=0$. From the fact that $x \tilde{R} x \dagger$ and that the congruence condition holds we have $y x \widetilde{R} y x \dagger$. But then $y x=0$ implies $y x \dagger=0$. Similarly from $y \tilde{L} y^{*}$ we obtain $y x+\tilde{L} y^{*} x \dagger$ but $y x \dagger=0$ implies $y^{*} x \dagger=0$.

(ii) Let $x, y, a, b \in S$ with $x \widetilde{H} y$ and $a \tilde{H} b$. Then $x, y \in \tilde{L}_{f}$ and $a, b \in \tilde{R}_{e}$ some $e, f \in U$. But $x a=0$ iff $f e=0$ iff $y b=0$ by (i). If $f e \neq 0$ then $x a \in \tilde{R}_{x} \cap \tilde{L}_{a}$ and $y b \in \tilde{R}_{y} \cap \tilde{L}_{b}$ by Lemma 2.12 and since $\tilde{R}_{x}=\tilde{R}_{y}$ and $\tilde{L}_{a}=\tilde{L}_{b}$ we have $x a \tilde{H} y b$.

(iii) Let $\tilde{H}_{a}=\widetilde{R}_{e} \cap \tilde{L}_{f}$ where $e, f \in U$ and let $x, y \in \tilde{H}_{a}$. If $f e=0$ then $x y=0$ so that $\tilde{H}_{a} \tilde{H}_{a}=\{0\}$. Otherwise $f e \neq 0$ so that $x y \neq 0$ and $x y \in \tilde{R}_{x} \cap \tilde{L}_{y}=\tilde{H}_{a}$ by Lemma 2.12 .

\section{Rees semigroups}

In this section we will obtain a Rees matrix representation for Rees semigroups, thereby obtaining a converse to the results of Section 1. Let $S$ be a $U$-semiabundant semigroup. Define the following subset:

$$
\operatorname{Reg}_{U}(S)=\{a \in S: \text { there exist } e, f \in U \text { such that } e L a R f\}
$$

Thus the elements of $\operatorname{Reg}_{U}(S)$ are regular, but not necessarily all regular elements of $S$ belong to $\operatorname{Reg}_{U}(S)$. If $a \in \operatorname{Reg}_{U}(S)$ then the set $V_{U}(a)=\left\{a^{\prime} \in V(a): a^{\prime} a, a a^{\prime} \in U\right\}$.

Lemma 3.1. (i) $\tilde{L} \cap\left(\operatorname{Reg}_{U}(S) \times \operatorname{Reg}_{U}(S)\right)=L \cap\left(\left(\operatorname{Reg}_{U}(S) \times \operatorname{Reg}_{U}(S)\right)\right.$.

(ii) $\tilde{R} \cap\left(\operatorname{Reg}_{U}(S) \times \operatorname{Reg}_{U}(S)\right)=R \cap\left(\left(\operatorname{Reg}_{U}(S) \times \operatorname{Reg}_{U}(S)\right)\right.$.

Proof. We will prove (i), the proof of (ii) is similar.

Let $a, b \in \operatorname{Reg}(S)$ with $a L b$. We indicated at the beginning of Section 2 that $L \subseteq \tilde{L}$ thus we have $a \tilde{L} b$.

Now let $a, b \in \operatorname{Reg}_{U}(S)$ with $a \tilde{L} b$. Let $a^{\prime} \in V_{U}(a)$ and $b^{\prime} \in V_{U}(b)$. Then $a\left(a^{\prime} a\right)=a$ implies $b\left(a^{\prime} a\right)=b$ since $a^{\prime} a \in U$. Similarly, $b\left(b^{\prime} b\right)=b$ implies $a\left(b^{\prime} b\right)=a$ whence $a L b$.

Note that the previous lemma holds in particular for elements of $U$. Define a relation $\hat{D}$ on $S$ as follows:

$(a, b) \in \hat{D}$ iff there exist elements $a^{*}, a \dagger, b^{*}, b \dagger$ such that $\left(a^{*}, b^{*}\right) \in D$ and $(a \dagger, b \dagger) \in D$.

Lemma 3.2. The relation $\hat{D}$ is an equivalence relation with $\hat{D} \subseteq \tilde{D}$. 
Proof. Straightforward.

Lemma 3.3. Let $S$ be $U$-semiabundant. If each $\tilde{H}$-class of $S$ contains an element of $\operatorname{Reg}_{u}(S)$ then:

$$
\tilde{D}=\hat{D}=\tilde{L} \circ \tilde{R}=\tilde{R} \circ \tilde{L}
$$

Proof. Let $(a, b) \in \tilde{D}$. Then there exist elements $a_{i}(1 \leqq i \leqq n)$ such that,

$$
a \tilde{L} a_{1} \tilde{R} a_{2} \ldots \tilde{L} a_{n} \tilde{R} b
$$

Choose $a_{i}^{\prime} \in \tilde{H} a_{i} \cap \operatorname{Reg}_{U}(S)(1 \leqq i \leqq n)$ so that

$$
a \tilde{L} a_{1}^{\prime} \tilde{R} a_{2}^{\prime} \ldots \tilde{L} a_{n}^{\prime} \tilde{R} b
$$

But by Lemma 3.1 we have $a \tilde{L} a_{1}^{\prime} R a_{2}^{\prime} \ldots L a_{n}^{\prime} \tilde{R} b$. But $a_{1}^{\prime} R a_{2}^{\prime} \ldots L a_{n}^{\prime}$ implies $a_{1}^{\prime} D a_{n}^{\prime}$ so there exists $c \in S$ such that $a_{1}^{\prime} L c R a_{n}^{\prime}$ whence $a \tilde{L} c \tilde{R} b$ giving $(a, b) \in \tilde{L} \circ \tilde{R}$. But $\tilde{L} \circ \tilde{R} \subseteq \tilde{D}$ thus $\tilde{D}=\tilde{L} \circ \tilde{R}$. By symmetry $\tilde{D}=\tilde{R} \circ \tilde{L}$.

Now let $(a, b) \in \tilde{D}$ then $(a, b) \in \tilde{L} \circ \tilde{R}$ so by a similar argument we may find an element $c \in \operatorname{Reg}_{v}(S)$ so that $a \tilde{L} c \tilde{R} b$. Now, $a^{*} \tilde{L} a \tilde{L} c$ so $a^{*} L c$. Similarly $b^{*} R c$. Whence $\left(a^{*}, b^{*}\right) \in D$. Likewise $(a \dagger, b \dagger) \in D$ giving $(a, b) \in \hat{D}$ so we have $\tilde{D} \subseteq \hat{D}$. By Lemma $3.2, \hat{D} \subseteq \tilde{D}$ which gives $\hat{D}=\tilde{D}$ as required.

The above lemma is a generalisation of a result of Asibong's [1] from the theory of abundant semigroup.

Lemma 3.4. If $U \backslash\{0\}$ is contained in a single D-class then $\operatorname{Reg}_{U}(S)$ intersects each $\tilde{H}$-class non-trivially.

Proof. Let $a \in S$. Then $k \tilde{R} a \tilde{L} h$ for some $k, h \in U \backslash\{0\}$. Now $(k, h) \in D$ so by Howie [6, Theorem II 3.5] there exists $c$ and $c^{\prime} \in V(c)$ such that $c^{\prime} c=h, c c^{\prime}=k$ with $c \in \widetilde{H}_{a}$. But then $c \in \operatorname{Reg}_{U}(S)$.

Lemma 3.5 In a primitive $U$-semiabundant semigroup with $U$ closed under basic products $e S e=\tilde{H}_{e}^{0}$ for all $e \in U \backslash\{0\}$.

Proof. Clearly $\tilde{H}_{e}^{0} \subseteq e S e$. Let $x \in e S e$ with $x \neq 0$. Then $e x=x=x e$. Now $x \tilde{L} f$ for some $f \in U$, but $x e=x$ implies $f e=f$. The set $U$ is closed under basic products so that $e f \in U$ and $e f \omega e$. Whence $e f=0$ or $e f=e$. If $e f=0$ then 


$$
0=x(e f)=(x e) f=x f=x
$$

contrary to our choice of $x$. Therefore we must have $e f=e$. Since $f e=f$ we have $e L f$ giving $x \tilde{L} e$ from the fact that $L \subseteq \tilde{L}$. We may similarly show that $x \tilde{R} e$. Thus we have $x \tilde{H} e$ as required.

We now come to our main result.

Theorem 3.6. Let $S$ be a Rees semigroup (with respect to $U$ ). Then $S$ is isomorphic to a Rees matrix semigroup $M^{0}(T ; I ; \Lambda ; P)$ where $T$ is a monoid with zero and $P$ is a $\Lambda \times I$-matrix such that each row of $P$ and each column contains at least one invertible element from $T$. Under the isomorphism $U$ is mapped bijectively onto $P(M)$.

Proof. Index the non-zero $\tilde{R}$-classes by a set $I$ and the non-zero $\tilde{L}$-classes by a set $\Lambda$ with $I \cap \Lambda=\{1\}$.

Let $e \in U \backslash\{0\}$ then $e S e$ is a monoid with zero and identity $e$. Put $T=e S e$. Note that by Lemma $3.5, e S e=\tilde{H}_{e}^{o}$.

In following the proof, it may be useful to draw a (generalised) eggbox diagram of $S$, with the $\tilde{R}$-classes being represented by the rows and the $\tilde{L}$-classes being represented by the columns, the intersection of the rows and the columns are precisely the $\tilde{H}$-classes. We label $\widetilde{H}_{e}=\tilde{H}_{11}$. The $\tilde{H}$-classes of $\widetilde{L}_{e}$ are labelled $\widetilde{H}_{i 1}(i \in I)$ and the $\tilde{H}$-classes of $\widetilde{R}_{e}$ are labelled $\tilde{H}_{1 \lambda}(\lambda \in \Lambda)$. By Lemma 3.4 , each $\tilde{H}$-class contains an element from $\operatorname{Reg}_{v}(S)$, pick $q_{\lambda} \in \tilde{H}_{1 \lambda} \cap \operatorname{Reg}_{U}(S)$ and $r_{i} \in \tilde{H}_{i 1} \cap \operatorname{Reg}_{U}(S)$. If $x \in S$ then $\lambda_{x}$ and $\rho_{x}$ will denote respectively left and right multiplication by the element $x$. We will show that the maps $\lambda r_{i}: \widetilde{H}_{e} \rightarrow \widetilde{H}_{i 1}$ and $p q_{\lambda}: \widetilde{H}_{i 1} \rightarrow \widetilde{H}_{i \lambda}$ are well-defined bijections. We prove this for the map $\lambda r_{i}$ the proof for $p q_{\lambda}$ is similar.

We begin by showing that the map $\lambda r_{i}$ is well defined. Let $f \in U$ such that $f \tilde{R} r_{i}$. Then we may choose (by Howie [6, Theorem II 3.5]) $r_{i}^{\prime} \in V_{U}\left(r_{i}\right)$ such that $r_{i} r_{i}^{\prime}=f$ and $r_{i}^{\prime} r_{i}=e$. If $x \tilde{H} e$ then $r_{i} x \neq 0$, for if $r_{i} x=0$ then $r_{i}^{\prime} r_{i} x=0$ whence $e x=0$. But $e x=x$ so that $x=0$, contradicting our choice of $x$. We also have that $r_{i} e \neq 0$ for $r_{i} e=r_{i} \neq 0$. Since both $r_{i} x \neq 0$ and $r_{i} e \neq 0$ and $x \widetilde{R} e$ we may apply the weak congruence condition and obtain $r_{i} x \tilde{R} r_{i} e=r_{i}$. We may similarly show that $r_{i} x \tilde{L} e x=x$. Thus we obtain $r_{i} x \in \tilde{H}_{i 1}$ as required.

The map $\lambda r_{i}$ is one-to-one, for if $x, y \in \tilde{H}_{e}$ and $r_{i} x=r_{i} y$ then $r_{i}^{\prime} r_{i} x=r_{i}^{\prime} r_{i} y$ giving $e x=e y$ whence $x=y$.

The map $\lambda r_{i}$ is onto for if $d \in \tilde{H}_{i 1}$ then $d \tilde{R} f$ so that by the weak congruence condition $r_{i}^{\prime} d \tilde{R} r_{i}^{\prime} f=r_{i}^{\prime}$ since $r_{i}^{\prime} d, r_{i}^{\prime} f \neq 0$. Also $r_{i}^{\prime} \tilde{L} f$ so $r_{i}^{\prime} d \tilde{L} f d=d$ since $r_{i}^{\prime} d, f d \neq 0$. This means that $r_{i}^{\prime} d \in \tilde{H}_{e}$. Furthermore $r_{i}\left(r_{i}^{\prime} d\right)=d$ since $f d=d$.

Now define a $\Lambda \times I$-matrix $P=\left(p_{\lambda i}\right)$ over $e S e$ by putting $p_{\lambda i}=q_{\lambda} r_{i}$. Note that by Lemma 2.12 either $q_{\lambda} r_{i}=0$ or $q_{\lambda} r_{i} \in \tilde{R} q_{\lambda} \cap \tilde{L}_{r_{i}}=\tilde{H}_{11}$. In either case $p_{\lambda i} \in e S e$. We need to show that for each $i \in I$ there exists $\lambda \in \Lambda$ such that $q_{\lambda} r_{i}$ is invertible in $e S e$. Since $S$ is $U$-semiabundant there exists $f \in U$ such that $f \tilde{R} r_{i}$ and we suppose $f \tilde{L} q_{\lambda}$ some $\lambda \in \Lambda$. By Howie [6, Theorem II 3.5] there exists $q_{\lambda}^{\prime} \in V\left(q_{\lambda}\right) \cap \tilde{H}_{r_{i}}$ and there exists $r_{i}^{\prime} \in V\left(r_{i}\right) \cap \tilde{H}_{q_{i}}$ such that

$$
q_{\lambda} q_{\lambda}^{\prime}=e, q_{\lambda}^{\prime} q_{\lambda}=f, r_{i}^{\prime} r_{i}=e \text { and } r_{i} r_{i}^{\prime}=f \text {. }
$$

Note first that $q_{\lambda} r_{i} \neq 0$, for $q_{\lambda} r_{i}=0$ implies $q_{\lambda}^{\prime} q_{\lambda} r_{i}=0$, that is $f r_{i}=0$ so that $r_{i}=0$ a 
contradiction. Thus we have $q_{\lambda} r_{i} \in \tilde{H}_{11}$. Similarly $r_{i}^{\prime} q_{\lambda}^{\prime} \in \tilde{H}_{11}$. It is straightforward to check that the element $r_{i}^{\prime} q_{\lambda}^{\prime}$ is the (group) inverse of $q_{\lambda} r_{i}$ in $e S e$ so that $p_{\lambda i}$ is invertible.

We may similarly show that for each $\lambda \in \Lambda$ there exists $i \in I$ such that the element $q_{\lambda} r_{i}$ is invertible.

Thus far we have shown, in particular, that $M^{0}(T ; I, \Lambda ; P)$ is a Rees matrix semigroup where $P$ is regular.

We may now define a map $\theta: S \rightarrow M^{0}(T ; I, \Lambda ; P)$ by putting $\theta(0)=0$ and if $s$ is a non-zero element, where $s \in \widetilde{H}_{i \lambda}$ and $x \in \widetilde{H}_{e}$ is the unique element such that $r_{i} x q_{\lambda}=s$, then define $\theta(s)=(i, x, \lambda)$. It is clear that $\theta$ is a bijection and easy to check that it is a homomorphism.

Finally note that $(\theta \mid U): U \rightarrow P(M)$, for if $f \in \tilde{H}_{i \lambda} \cap U$ then $r_{i}\left(r_{i}^{\prime} q_{\lambda}^{\prime}\right) q_{\lambda}=f^{2}$ so that $\theta(f)=\left(i, r_{i}^{\prime} q_{\lambda}^{\prime}, \lambda\right)=\left(i, p_{\lambda i}^{-1}, \lambda\right)$.

\section{Corollary 3.7.}

$$
\theta\left(\operatorname{Reg}_{U}(S)\right)=I \times G(e S e)^{0} \times \Lambda
$$

Proof. Let $r \in \operatorname{Reg}_{U}(S)$ with $h R r L g$ where $h, g \in U$ and let $\theta(r)=(i, x, \lambda)$ so that $r=r_{i} x q_{\lambda}$. By Howie [6, Theorem II 3.5] we may find inverses $r^{\prime} \in V(r), q_{\lambda}^{\prime} \in V\left(q_{\lambda}\right)$ and $r_{i}^{\prime} \in V\left(r_{i}\right)$ such that

$$
r r^{\prime}=h, r^{\prime} r=g, r_{i} r_{i}^{\prime}=h, r_{i}^{\prime} r_{i}=e, q_{\lambda} q_{\lambda}^{\prime}=e \text { and } q_{\lambda}^{\prime} q_{\lambda}=g .
$$

It is easy to check that $x=r_{i}^{\prime} r q_{\lambda}^{\prime}$ and that it has the (group) inverse $q_{\lambda} r^{\prime} r_{i}$ in $e S e$.

Now let $x$ be invertible in $e S e$ with inverse $x^{-1}$. Then the element $r_{i} x q_{\lambda}$ for any $r_{i}$ and $q_{\lambda}$ belongs to $\operatorname{Reg}{ }_{U}(S)$ : for choosing $q_{\lambda}^{\prime}$ and $r_{i}^{\prime}$ as above (with $r$ replaced by $r_{i} x q_{\lambda}$ ) it is straightforward to check that $q_{i}^{\prime} x^{-1} r_{i}^{\prime} \in V_{v}\left(r_{i} x q_{\lambda}\right)$.

Corollary 3.8. The matrix $P$ has the property that every non-zero entry is invertible iff $\operatorname{Reg}_{U}(S)$ is a subsemigroup of $S$.

Proof. If all non-zero entries of $P$ are invertible and $(i, x, \lambda),(j, y, \mu) \in \operatorname{Reg}_{v}(\theta(S))$ then by the previous corollary $x$ and $y$ are invertible in eSe. Also $p_{\lambda j}=0$ or is itself invertible so that in either case $x p_{\lambda j} y \in G(e S e)^{0}$ whence $\left.(i, x, \lambda)(j, y, \mu) \in \operatorname{Reg}_{U}(S)\right)$. Thus $\operatorname{Reg}_{U}(S)$ is a subsemigroup of $S$.

Conversely suppose that $\operatorname{Reg}_{U}(S)$ is a subsemigroup and let $q_{\lambda} r_{i} \neq 0$. Then $q_{\lambda} r_{i} \in \operatorname{Reg}{ }_{U}(S)$ and $q_{\lambda} r_{i} \in e S e$ so that $q_{\lambda} r_{i} \in \widetilde{H}_{e}$ whence $q_{\lambda} r_{i} H e$ by Lemma 3.1. Thus $q_{\lambda} r_{i}$ is invertible.

The following corollary is now immediate from Corollary 3.8 and Lemma 1.9.

Corollary 3.9. (i) A Rees semigroup is isomorphic to a Rees matrix semigroup over a monoid with zero adjoined iff it satisfies the congruence condition.

(ii) A Rees semigroup is isomorphic to a Rees matrix semigroup over a monoid with zero 
adjoined and in which every non-zero entry of $P$ is invertible iff it satisfies the congruence condition and $\operatorname{Reg}_{v}(S)$ is a subsemigroup.

The semigroups $S$ of Corollary 3.9 (ii) may characterised as follows: $S$ is a semigroup with zero 0 having a subsemigroup $T$ which is completely 0 -simple (with $0 \in T$ ) furthermore $S$ is $E(T)$-semiabundant and satisfies the congruence condition (or equivalently $e S e$ is a semigroup with zero adjoined for some non-zero element $e \in E(T)$ ). This provides an alternative characterisation of the semigroups of Theorem 3.4 [8].

A $U$-semiabundant semigroup $S$ is called reduced if $\omega^{r}=\omega^{l}$ on $U$.

Lemma 3.10. Let $S$ be $U$-semiabundant with $U$ closed under basic products. Then $S$ is reduced iff each $\tilde{L}$-class and each $\tilde{R}$-class contains a unique element from $U$.

Proof. Let $S$ be reduced and suppose that $e \tilde{L} f$ where $e, f \in U$. By Lemma $3.1 e L f$ so that $e f=e$ and $f e=f$. But $e f=e$ iff $e \omega^{l} f$. By assumption this gives $e \omega^{r} f$, that is $f e=e$. But then $e=f e=f$.

Conversely let each $\tilde{L}$-class and each $\tilde{R}$-class contain a unique element from $U$. If $e, f \in U$ with $e \omega^{r} f$ then $e f \in U$, since $U$ is closed under basic products and ef Re. But this implies ef $R e$, so that $e f=e$ whence $e \omega^{l} f$. The inclusion in the other direction is similar.

The following is immediate:

Lemma 3.11. A Rees matrix semigroup is reduced iff each row and each column of $P$ contains a unique invertible element.

To conclude this section we give some well-known results in terms of our approach:

Proposition 3.12. Let $S$ be a Rees semigroup with $e \in U \backslash\{0\}$ then,

(i) $S$ is abundant iff $e$ Se is abundant.

(ii) $S$ is regular iff eSe is regular.

(iii) $S$ is inverse iff $S$ is reduced, eSe is inverse and $\operatorname{Reg}_{U}(S)$ is a subsemigroup.

Proof. (i) Proposition 2.11 and Lemma 2.10 [9].

(ii) Theorem 4 (13].

(iii) Theorem $6[13]$.

Finally we note that the results of this paper may be generalised to incorporate the results of Steinfeld [16]. A special case of this more general result extends that obtained by Batbedat and Reilly [3]: they consider square Rees matrix semigroups over monoids with zero adjoined having locally regular sandwich matrices. 
Acknowledgement. I would like to thank the referee for making a number of comments which led to an improvement in the presentation of this paper.

\section{REFERENCES}

1. U. Asıbong-IBE, The structure of type A $\omega$-semigroups (D.Phil. Thesis, York, 1981).

2. C. M. de Barros, Sur les catégories ordonnés régulières, Cahiers Topologie Géom. Différentielle Catégoriques 11 (1969), 23-55.

3. A. Batbedat and N. R. Reilly, Categories and biunary semigroups, Simon Stevin 56 (1982), 181-209.

4. A. El-QAllali, Structure theory for abundant and related semigroups (D.Phil. Thesis, York, 1980).

5. J. B. Fountain, Abundant semigroups, Proc. London Math. Soc. (3) 44 (1982), 103-129.

6. J. M. HowIE, An Introduction to the Theory of Semigroups (Academic Press, London, 1976).

7. G. Lallement and M. Petrich, Décompositions I-matricielles d'un demi-groupe, J. Math. Pures Appl. 45 (1966), 67-117.

8. G. Lallement and M. Petrich, A generalisation of the Rees theorem in semigroups, Acta. Sci. Math. (Szeged) 30 (1969), 113-132.

9. M. V. Lawson, Abundant Rees matrix semigroups, J. Austral. Math. Soc. Ser. A 42 (1987), $132-142$.

10. M. V. Lawson, Primitive U-semiabundant semigroups, in preparation.

11. M. V. Lawson, Semigroups and ordered categories I, submitted.

12. M. V. Lawson, Semigroups and ordered categories II, in preparation.

13. L. MárKi, On locally regular Rees matrix semigroups, Acta. Sci. Math. (Szeged) 37 (1975), 95-102.

14. J. Meakin, The Rees construction in regular semigroups, Colloq. Math. Soc. János Bolyai 39 (1981), 115-156.

15. D. Rees, On semi-groups, Proc. Cambridge Philos. Soc. 36 (1940), 387-400.

16. O. Steinfeld, On a generalisation of completely 0-simple semigroups, Acta. Sci. Math. (Szeged) 28 (1967), 135-145.

17. A. SuschKewitsch, Über die endlichen Gruppen ohne das Gesetz der eindeutigen Umkehrbarkeit, Math. Ann. 99 (1928), 30-50.

Fachbereich Mathematik AG5

TeChNisChe Hochschule

D.6100 DARMSTADT

SCHLOSSGARTENSTR. 7

West Germany
Current Address:

School of Mathematics

University College of North Wales

Dean Street, Bangor, Gwynedd Ll57 IUT

WALES 